\title{
Distance measure between intuitionistic fuzzy sets and its application to pattern recognition
}

\author{
Jin Han Park ${ }^{1}$, Ki Moon Lim ${ }^{1}$ and Young Chel Kwun ${ }^{2}$ \\ ${ }^{1}$ Division of Mathematical Sciences, Pukyong National University, Pusan 608-737, Korea \\ 2 Department of Mathematics, Dong-A University, Pusan 604-714, Korea
}

\begin{abstract}
In this paper, we propose new method to calculate the distance between intuitionistic fuzzy sets (IFSs) based on the three dimensional representation of IFSs and analyze the relations of similarity measure and distance measure of IFSs. Finally, we apply the proposed measures to pattern recognitions.
\end{abstract}

Key words : Intuitionistic fuzzy sets, Distance measures, Similarity measures, Pattern recognition.

\section{Introduction}

Fuzzy set theory, proposed by Zadeh [21], has showed successful applications in various fields. In fuzzy set theory, the membership of an element to a fuzzy set is a single value between 0 and 1 . But in reality, it may not always be certain that the degree of nonmembership of an element in a fuzzy set is just equal to 1 minus the degree of membership. That is to say, there may be some hesitation degree. So, as a generalization of fuzzy sets, the concept of intuitionistic fuzzy sets (IFSs) was introduced by Atanassov [1, 2, 3]. Burillo and Bustince [5] pointed out that this notion coincides with the notion of vague sets proposed by Gau and Buehere [9]. These IFSs describe the objective world more realistic, practical and accurate. They have been widely studied and applied in various areas, such as logic programming [4], decision making [7, 15], pattern recognition $[10,11,12,13,14,18]$ and medical diagnosis $[8,17]$, and seem to have more popular than fuzzy sets technology.

As important contents in fuzzy mathematics, similarity measure and distance measure between IFSs have been attracted many researchers. Chen [6], Chen and Tan [7] proposed several similarity measures for measuring the degree of similarity of vague sets. Li and Cheng [11] and discussed similarity measures between IFSs and showed how these measures may be used in pattern recognition problems. However, Li and Cheng's similarity measures

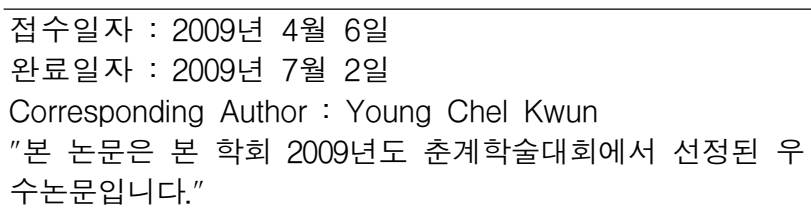

may not be effective in some cases. To overcome the drawbacks of Li and Cheng's methods, Liang and Shi [13] and Mitchell [14] made some modifications, respectively. Szmidt and Kacprzky [16] proposed four distance measures between IFSs that were in some extent based the geometrical interpretation of IFSs, and have some good geometric properties. Hung and Yang [10] proposed another method to calculate the distance between IFSs based on the Hausdorff distance. They used this distance to generate several similarity measures between IFSs that are suited to be used in linguistic variables. Wang and Xin [19] proposed several distance measures between IFSs and applied those measures to pattern recognitions.

In this paper, we propose new method to calculate the distance between IFSs based on the three dimensional representation of IFSs and analyze the relations of similarity measure and distance measure of IFSs. Finally, we apply the proposed measures to pattern recognitions.

\section{Basic notions of IFSs}

In the following, we firstly recall basic notions and definitions of IFSs which can be found in $[1,2,3]$.

Let $X$ be the universe of discourse. An IFS $A$ in $X$ is an object having the form

$$
A=\left\{\left(x, \mu_{A}(x), \nu_{A}(x)\right): x \in X\right\}
$$

where $\mu_{A}, \nu_{A}: X \rightarrow[0,1]$ denote, respectively, membership and non-membership functions of $A$ with the condi- 
tion $0 \leq \mu_{A}(x)+\nu_{A}(x) \leq 1$ for any $x \in X$. Let $\operatorname{IFS}(X)$ denote the set of all IFSs in $X$.

Obviously, any fuzzy set $A$ in $X$ may be represented as the following IFS: $A=\left\{\left(x, \mu_{A}(x), 1-\mu_{A}(x)\right): x \in X\right\}$.

For each $A \in \operatorname{IFS}(X)$, we call

$$
\pi_{A}(x)=1-\mu_{A}(x)-\nu_{A}(x)
$$

the intuitionistic index of $x$ in $A$. It is hesitancy degree of $x$ to $A$ (see, $[2,3]$ ). Obviously, $0 \leq \pi_{A}(x) \leq 1$ for each $x \in X$.

As Szmidt and Kacprzky's work [16], considering the term of hesitancy degree, an IFS $A$ can be expressed as

$$
A=\left\{\left(\mu_{A}(x), \nu_{A}(x), \pi_{A}(x)\right): x \in X\right\} .
$$

If $A$ is an ordinary fuzzy set, then $\pi_{A}(x)=0$ for each $x \in X$. It means that third parameter $\pi_{A}(x)$ can not be casually omitted if $A$ is a general IFS, not an ordinary fuzzy set. Therefore, this representation of IFSs will be a point of departure for considering new method to calculate the distance between IFSs.

For $A, B \in \operatorname{IFS}(X)$, Atanassov [2] defined the notion of inclusion as follows:

$$
\begin{aligned}
A \subseteq B \Leftrightarrow & \mu_{A}(x) \leq \mu_{B}(x) \text { and } \nu_{A}(x) \geq \nu_{B}(x) \\
& \text { for all } x \in X .
\end{aligned}
$$

As above-mentioned, we can not omit the third parameter (hesitancy degree) in the representation of IFSs and then redefine the notion of containment as follows:

$$
\begin{aligned}
A \subseteq B \Leftrightarrow & \mu_{A}(x) \leq \mu_{B}(x), \nu_{A}(x) \geq \nu_{B}(x) \\
& \text { and } \pi_{A}(x) \geq \pi_{B}(x) \text { for all } x \in X .
\end{aligned}
$$

Measuring the similarity between IFSs is important in pattern recognition research. Some methods have previously been advanced to caculate the degree of similarity between IFSs $[10,11,13,14]$. In the following, we shall review these similarity measures. In the study of the similarity between IFSs, Li and Cheng [11] and Mitchell [14] introduced the following definition.

Definition 1. A function $S: \operatorname{IFSs}(X) \times \operatorname{IFSs}(X) \rightarrow$ $[0,1]$ is called similarity measure of IFSs if $S$ satisfies the following properties: for any $A, B, C \in \operatorname{IFSs}(X)$,

(SP1) $0 \leq S(A, B) \leq 1$;

(SP2) $S(A, B)=1$ if and only if $A=B$;

(SP3) $S(A, B)=S(B, A)$;

(SP4) If $A \subseteq B \subseteq C$, then $S(A, C) \leq S(A, B)$ and $S(A, C) \leq S(B, C)$.

Distance measure is another measure in fuzzy set theory, and is the measure of difference between IFSs. Wang and Xin [19] defined the axiom definition of it as follows:

Definition 2. A function $d: \operatorname{IFSs}(X) \times \operatorname{IFSs}(X) \rightarrow$ $[0,1]$ is called distance measure of IFSs if $d$ satisfies the following properties: for any $A, B, C \in \operatorname{IFSs}(X)$,
(DP1) $0 \leq d(A, B) \leq 1$;

(DP2) $d(\bar{A}, B)=0$ if and only if $A=B$;

(DP3) $d(A, B)=d(B, A)$;

(DP4) If $A \subseteq B \subseteq C$, then $d(A, C) \geq d(A, B)$ and $d(A, C) \geq d(B, C)$.

Distance measure is a term that describes the difference between IFSs, and can be considered as a dual concept of similarity measure. Many measures of distance measures between IFSs have been proposed and researched in recent years.

Assume that there are two IFSs $A$ and $B$ in $X=$ $\left\{x_{1}, x_{2}, \ldots, x_{n}\right\}$.

Li and Cheng [11] proposed the distance $d_{d}^{p}$ between IFSs $A$ and $B$ as follows:

$$
d_{d}^{p}(A, B)=\frac{1}{\sqrt[p]{n}} \sqrt[p]{\sum_{i=1}^{n}\left|m_{A}(i)-m_{b}(i)\right|^{p}}
$$

where $m_{A}(i)=\left(\mu_{A}\left(x_{i}\right)+1-\nu_{A}\left(x_{i}\right)\right) / 2, m_{B}(i)=$ $\left(\mu_{B}\left(x_{i}\right)+1-\nu_{B}\left(x_{i}\right)\right) / 2$ and $1 \leq p<\infty$. Clearly, $S_{d}^{p}$ $\left(=1-d_{d}^{p}\right)$ is similarity measure since distance and similarity measure are dual concepts. This measure is not only easily interpreted in geometry but also easily calculated in practical cases.

Liang and Shi [13] proposed the distance $d_{e}^{p}$ between IFSs $A$ and $B$ as follows:

$$
d_{e}^{p}(A, B)=\frac{1}{\sqrt[p]{n}} \sqrt[p]{\sum_{i=1}^{n}\left|\phi_{t A B}(i)-\phi_{f A B}(i)\right|^{p}}
$$

where $\phi_{t A B}(i)=\left|\mu_{A}\left(x_{i}\right)-\mu_{B}\left(x_{i}\right)\right| / 2, \phi_{f A B}(i)=\mid(1-$ $\left.\nu_{A}\left(x_{i}\right)\right) / 2-\left(1-\nu_{B}\left(x_{i}\right)\right) / 2 \mid$ and $1 \leq p<\infty$. Then $S_{e}^{p}(A, B)\left(=1-d_{e}^{p}(A, B)\right)$ is similarity measure between $A$ and $B$. To get more information on IFSs, Liang and Shi [13] used the middle point $m_{A}(i)$ to partition the interval $\left[\mu_{A}\left(x_{i}\right), 1-\nu_{A}\left(x_{i}\right)\right]$ into two subintervals $\left[\mu_{A}\left(x_{i}\right), m_{A}(i)\right]$ and $\left[m_{A}(i), 1-\nu_{A}\left(x_{i}\right)\right]$. Then they considered the middle points, say $m_{A 1}(i)$ and $m_{A 2}(i)$, of these two subintervals. That is, $m_{A 1}(i)=\frac{\mu_{A}\left(x_{i}\right)+m_{A}(i)}{2}, m_{A 2}(i)=$ $\frac{m_{A}(i)+1-\nu_{A}\left(x_{i}\right)}{2}$. By the same way, $m_{B 1}(i)$ and $m_{B 2}(i)$ are the middle points of the subintervals $\left[\mu_{B}\left(x_{i}\right), m_{B}(i)\right]$ and $\left[m_{B}(i), 1-\nu_{B}\left(x_{i}\right)\right]$, respectively. Thus, they proposed the distance between $A$ and $B$ as follows:

$$
d_{s}^{p}(A, B)=\frac{1}{\sqrt[p]{n}} \sqrt[p]{\sum_{i=1}^{n}\left(\phi_{s 1}(i)-\phi_{s 2}(i)\right)^{p}}
$$

where $\phi_{s 1}(i)=\left|m_{A 1}(i)-m_{B 1}(i)\right| / 2$ and $\phi_{s 2}(i)=$ $\left|m_{A 2}(i)-m_{B 2}(i)\right| / 2$. Hence, they used $S_{s}^{p}(A, B)(=$ $\left.1-d_{s}^{p}(A, B)\right)$ to measure the degree of similarity between $A$ and $B$. In comparison of $S_{d}^{p}$ and $S_{s}^{p}$, the formula of $S_{d}^{p}$ is simpler than $S_{s}^{p}$, but $S_{s}^{p}$ can catch more information in IFSs than $S_{d}^{p}$. 
Mitchell [14] adopted a statistical approach and interpreted IFSs as ensembles of ordered fuzzy sets to modify $\mathrm{Li}$ and Cheng's similarity measure. Let $\rho_{\mu}(A, B)$ and $\rho_{\nu}(A, B)$ denote the similaritys between the low membership functions $\mu_{A}$ and $\mu_{B}$ and between the high membership functions $1-\nu_{A}$ and $1-\nu_{B}$, respectively, as follows:

$$
\begin{aligned}
\rho_{\mu}(A, B) & =S\left(\mu_{A}, \mu_{B}\right) \\
& =1-\frac{1}{\sqrt[p]{n}} \sqrt[p]{\sum_{i=1}^{n}\left|\mu_{A}\left(x_{i}\right)-\mu_{B}\left(x_{i}\right)\right|^{p}} \\
\rho_{\nu}(A, B) & =S\left(1-\nu_{A}, 1-\nu_{B}\right) \\
& =1-\frac{1}{\sqrt[p]{n}} \sqrt[p]{\sum_{i=1}^{n}\left|\nu_{A}\left(x_{i}\right)-\nu_{B}\left(x_{i}\right)\right|^{p}} .
\end{aligned}
$$

He then defined the modified similarity measure between IFSs $A$ and $B$ with

$$
S_{\mathrm{mod}}(A, B)=\frac{1}{2}\left(\rho_{\mu}(A, B)+\rho_{\nu}(A, B)\right) .
$$

Hung and Yang [10] used the idea of Hausdorff distance to define the distance between IFSs $A$ and $B$ as follows:

$$
\begin{gathered}
d_{H}(A, B)=\frac{1}{n} \sum_{i=1}^{n} \max \left\{\left|\mu_{A}\left(x_{i}\right)-\mu_{B}\left(x_{i}\right)\right|,\right. \\
\left.\left|\nu_{A}\left(x_{i}\right)-\nu_{B}\left(x_{i}\right)\right|\right\}
\end{gathered}
$$

and used the distance $d_{H}$ to generate three similarity measures:

$$
\begin{aligned}
& S_{l}(A, B)=1-d_{H}(A, B), \\
& S_{e}(A, B)=\frac{\exp \left(-d_{H}(A, B)\right)-\exp (-1)}{1-\exp (-1)}, \\
& S_{c}(A, B)=\frac{1-d_{H}(A, B)}{1+d_{H}(A, B)} .
\end{aligned}
$$

Wang and Xin [19] proposed the distance measures between IFSs $A$ and $B$ as follows:

$$
\begin{aligned}
d_{\mathrm{wx} 1}( & A, B) \\
= & \frac{1}{n} \sum_{i=1}^{n}\left(\frac{\left|\mu_{A}\left(x_{i}\right)-\mu_{B}\left(x_{i}\right)\right|+\left|\nu_{A}\left(x_{i}\right)-\nu_{B}\left(x_{i}\right)\right|}{4}\right. \\
& \left.+\frac{\max \left\{\left|\mu_{A}\left(x_{i}\right)-\mu_{B}\left(x_{i}\right)\right|,\left|\nu_{A}\left(x_{i}\right)-\nu_{B}\left(x_{i}\right)\right|\right\}}{2}\right)
\end{aligned}
$$

and

$$
d_{\mathrm{wx} 2}(A, B)=\frac{1}{\sqrt[p]{n}} \sqrt[p]{\sum_{i=1}^{n}\left(\phi_{\mu}(i)-\phi_{\nu}(i)\right)^{p}},
$$

where $\phi_{\mu}(i)=\left|\mu_{A}\left(x_{i}\right)-\mu_{B}\left(x_{i}\right)\right| / 2$ and $\phi_{\nu}(i)=$ $\left|\nu_{A}\left(x_{i}\right)-\nu_{B}\left(x_{i}\right)\right| / 2$.

\section{New distance measures between IFSs}

In this section, based on the three dimensional representation of IFSs, we propose new method to calculate the distance between IFSs by adding the term of hesitancy to Wang and Xin's formula.

For two IFSs $A$ and $B$ in $X=\left\{x_{1}, x_{2}, \ldots, x_{n}\right\}$, denote

$d_{1}(A, B)$

$$
\begin{aligned}
= & \frac{1}{4 n} \sum_{i=1}^{n}\left(\left|\mu_{A}\left(x_{i}\right)-\mu_{B}\left(x_{i}\right)\right|+\left|\nu_{A}\left(x_{i}\right)-\nu_{B}\left(x_{i}\right)\right|\right. \\
& +\left|\pi_{A}\left(x_{i}\right)-\pi_{B}\left(x_{i}\right)\right|+2 \max \left\{\left|\mu_{A}\left(x_{i}\right)-\mu_{B}\left(x_{i}\right)\right|,\right. \\
& \left.\left.\left|\nu_{A}\left(x_{i}\right)-\nu_{B}\left(x_{i}\right)\right|,\left|\pi_{A}\left(x_{i}\right)-\pi_{B}\left(x_{i}\right)\right|\right\}\right) .
\end{aligned}
$$

Then we have the following theorem.

Theorem 1. $d_{1}(A, B)$ is the degree of distance between two IFSs $A$ and $B$ in $X=\left\{x_{1}, x_{2}, \ldots, x_{n}\right\}$.

Proof. It is easy to see that $d_{1}(A, B)$ satisfies the properties (DP1)-(DP3) of Definition 2. We therefore only prove that $d_{1}(A, B)$ satisfies the property (DP4).

Let $A \subseteq B \subseteq C, A, B, C \in \operatorname{IFSs}(X)$. Then $\mu_{A}\left(x_{i}\right) \leq \mu_{B}\left(x_{i}\right) \mu_{C}\left(x_{i}\right), \nu_{A}\left(x_{i}\right) \geq \nu_{B}\left(x_{i}\right) \geq \nu_{C}\left(x_{i}\right)$ and $\pi_{A}\left(x_{i}\right) \geq \pi_{B}\left(x_{i}\right) \geq \pi_{C}\left(x_{i}\right)$ for any $x_{i} \in X$. It follows that

$$
\begin{aligned}
& \left|\mu_{A}\left(x_{i}\right)-\mu_{C}\left(x_{i}\right)\right| \geq\left|\mu_{A}\left(x_{i}\right)-\mu_{B}\left(x_{i}\right)\right|, \\
& \left|\nu_{A}\left(x_{i}\right)-\nu_{C}\left(x_{i}\right)\right| \geq\left|\nu_{A}\left(x_{i}\right)-\nu_{B}\left(x_{i}\right)\right|, \\
& \left|\pi_{A}\left(x_{i}\right)-\pi_{C}\left(x_{i}\right)\right| \geq\left|\pi_{A}\left(x_{i}\right)-\pi_{B}\left(x_{i}\right)\right| .
\end{aligned}
$$

So, we have

$$
\begin{aligned}
& \left|\mu_{A}\left(x_{i}\right)-\mu_{C}\left(x_{i}\right)\right|+\left|\nu_{A}\left(x_{i}\right)-\nu_{C}\left(x_{i}\right)\right| \\
& \quad+\left|\pi_{A}\left(x_{i}\right)-\pi_{C}\left(x_{i}\right)\right|+2 \max \left\{\left|\mu_{A}\left(x_{i}\right)-\mu_{C}\left(x_{i}\right)\right|,\right. \\
& \left.\quad\left|\nu_{A}\left(x_{i}\right)-\nu_{C}\left(x_{i}\right)\right|,\left|\pi_{A}\left(x_{i}\right)-\pi_{C}\left(x_{i}\right)\right|\right\} \\
& \geq\left|\mu_{A}\left(x_{i}\right)-\mu_{B}\left(x_{i}\right)\right|+\left|\nu_{A}\left(x_{i}\right)-\nu_{B}\left(x_{i}\right)\right| \\
& \quad+\left|\pi_{A}\left(x_{i}\right)-\pi_{B}\left(x_{i}\right)\right|+2 \max \left\{\left|\mu_{A}\left(x_{i}\right)-\mu_{B}\left(x_{i}\right)\right|,\right. \\
& \left.\quad\left|\nu_{A}\left(x_{i}\right)-\nu_{B}\left(x_{i}\right)\right|,\left|\pi_{A}\left(x_{i}\right)-\pi_{B}\left(x_{i}\right)\right|\right\}
\end{aligned}
$$

and then we can get $d_{1}(A, C) \geq d_{1}(A, B)$. By the same reason, we can get $d_{1}(A, C) \geq d_{1}(B, C)$. Thus the property (DP4) is obtained.

Example 1. Let $A$ and $B$ be two IFSs in $X=\left\{x_{1}, x_{2}\right.$, $\left.x_{3}\right\}$ given by $A=\{(0.3,0.6),(0.5,0.4),(0.7,0.1)\}$ and $B=\{(0.4,0.6),(0.6,0.3),(0.5,0.2)\}$. Then, by the definition of $d_{1}$, the distance between $A$ and $B$ is

$d_{1}(A, B)$

$$
\begin{aligned}
= & \frac{1}{12} \sum_{i=1}^{3}\left(\left|\mu_{A}\left(x_{i}\right)-\mu_{B}\left(x_{i}\right)\right|+\left|\nu_{A}\left(x_{i}\right)-\nu_{B}\left(x_{i}\right)\right|\right. \\
+ & \left|\pi_{A}\left(x_{i}\right)-\pi_{B}\left(x_{i}\right)\right|+2 \max \left\{\left|\mu_{A}\left(x_{i}\right)-\mu_{B}\left(x_{i}\right)\right|,\right. \\
& \left.\left.\quad\left|\nu_{A}\left(x_{i}\right)-\nu_{B}\left(x_{i}\right)\right|,\left|\pi_{A}\left(x_{i}\right)-\pi_{B}\left(x_{i}\right)\right|\right\}\right) \\
= & 0.112
\end{aligned}
$$


Usually, the weight of the element $x_{i} \in X$ should be taken into account, so we present the following weighted distance measures between IFSs.

Assume the weight of the element $x_{i} \in X=\left\{x_{1}\right.$, $\left.x_{2}, \ldots, x_{n}\right\}$ is $\omega_{i}(i=1,2, \ldots, n)$, where $0 \leq \omega_{i} \leq 1$. Denote

$$
\begin{aligned}
d_{\omega}(A, B) & \\
= & \frac{1}{4} \sum_{i=1}^{n} \omega_{i}\left(\left|\mu_{A}\left(x_{i}\right)-\mu_{B}\left(x_{i}\right)\right|+\left|\nu_{A}\left(x_{i}\right)-\nu_{B}\left(x_{i}\right)\right|\right. \\
+ & \left|\pi_{A}\left(x_{i}\right)-\pi_{B}\left(x_{i}\right)\right|+2 \max \left\{\left|\mu_{A}\left(x_{i}\right)-\mu_{B}\left(x_{i}\right)\right|,\right. \\
& \left.\left.\left|\nu_{A}\left(x_{i}\right)-\nu_{B}\left(x_{i}\right)\right|,\left|\pi_{A}\left(x_{i}\right)-\pi_{B}\left(x_{i}\right)\right|\right\}\right) / \sum_{i=1}^{n} \omega_{i} .
\end{aligned}
$$

Then we have the following theorem.

Theorem 2. $d_{\omega}(A, B)$ is the degree of distance between two IFSs $A$ and $B$ in $X=\left\{x_{1}, x_{2}, \ldots, x_{n}\right\}$.

Proof. The proof is similar to that of Theorem 1 .

Remark 1. Obviously, if $w_{i}=1 / n(i=1,2, \ldots, n)$, $d_{\omega}(A, B)$ becomes $d_{1}(A, B)$. So, $d_{1}(A, B)$ is a special case of $d_{\omega}(A, B)$.

Now, we propose another distance measure between IFSs.

For IFSs $A$ and $B$ in $X=\left\{x_{1}, x_{2}, \ldots, x_{n}\right\}$. Let $\phi_{\mu_{A B}}(i)=\left|\mu_{A}\left(x_{i}\right)-\mu_{B}\left(x_{i}\right)\right| / 2, \phi_{\nu_{A B}}(i)=\mid \nu_{A}\left(x_{i}\right)-$ $\nu_{B}\left(x_{i}\right)\left|/ 2, \phi_{\pi_{A B}}(i)=\right| \pi_{A}\left(x_{i}\right)-\pi_{B}\left(x_{i}\right) \mid / 2$ and $x_{i} \in X$. Denote

$d_{2}^{p}(A, B)=\frac{1}{\sqrt[p]{n}} \sqrt[p]{\sum_{i=1}^{n}\left(\phi_{\mu_{A B}}(i)+\phi_{\nu_{A B}}(i)+\phi_{\pi_{A B}}(i)\right)^{p}}$,

where $1 \leq p<\infty$.

Theorem 3. $d_{2}^{p}(A, B)$ is the degree of distance between two IFSs $A$ and $B$ in $X=\left\{x_{1}, x_{2}, \ldots, x_{n}\right\}$.

Proof. The proof is similar to that of Theorem 1.

Example 2. Let $A$ and $B$ be two IFSs defined in Example 1 . Then by the definition of $d_{2}^{1}$, the distance between $A$ and $B$ is

$$
\begin{aligned}
d_{2}^{1}(A, B)= & \frac{1}{6} \sum_{i=1}^{3}\left[\left|\mu_{A}\left(x_{i}\right)-\mu_{B}\left(x_{i}\right)\right|+\mid \nu_{A}\left(x_{i}\right)\right. \\
& \left.\quad-\nu_{B}\left(x_{i}\right)|+| \pi_{A}\left(x_{i}\right)-\pi_{B}\left(x_{i}\right) \mid\right] \\
= & 0.100 .
\end{aligned}
$$

Remark 2. By the same way, we can get its weighted distance measure as the weighted distance measure defined above.

It is well known that similarity measures can be generated from distance measures. Therefore, we may use the proposed distance measure to define a similarity measure.
Let $f$ be a monotone decreasing function and $d$ be the proposed distance measure. Since $0 \leq d(A, B) \leq 1$,

$$
f(1) \leq f(d(A, B)) \leq f(0) .
$$

This implies

$$
0 \leq \frac{f(d(A, B))-f(1)}{f(0)-f(1)} \leq 1 .
$$

Thus, we may define the similarity measure between IFSs $A$ and $B$ as follows:

$$
S(A, B)=\frac{f(d(A, B))-f(1)}{f(0)-f(1)} .
$$

Obviously, $S(A, B)$ satisfies properties (SP1)-(SP4).

Next, we follow the way of Hung and Yang [10] to choose

$$
f(x)=1-x \text { and } \frac{1}{1+x} .
$$

Then the corresponding similarity measures between $A$ and $B$ are given by

$$
\begin{aligned}
& S_{1}(A, B)=1-d_{1}(A, B), \\
& S_{2}(A, B)=1-d_{2}^{p}(A, B), \\
& S_{3}(A, B)=\frac{1-d_{1}(A, B)}{1+d_{1}(A, B)}, \\
& S_{4}(A, B)=\frac{1-d_{2}^{p}(A, B)}{1+d_{2}^{p}(A, B)} .
\end{aligned}
$$

\section{Numerical examples}

To illustrate the proposed similarity measures are reasonable, we borrow several examples from Liang and Shi [13].

Example 3. [13] Assume that there are three patterns denoted with IFSs in $X=\left\{x_{1}, x_{2}, x_{3}\right\}$. The three patterns are denoted as follows:

$$
\begin{aligned}
& A_{1}=\left\{\left(x_{1}, 0.3,0.3\right),\left(x_{2}, 0.2,0.2\right),\left(x_{3}, 0.1,0.1\right)\right\} \\
& A_{2}=\left\{\left(x_{1}, 0.2,0.2\right),\left(x_{2}, 0.2,0.2\right),\left(x_{3}, 0.2,0.2\right)\right\} \\
& A_{3}=\left\{\left(x_{1}, 0.4,0.4\right),\left(x_{2}, 0.4,0.4\right),\left(x_{3}, 0.4,0.4\right)\right\} .
\end{aligned}
$$

Assume that a sample $B=\left\{\left(x_{1}, 0.3,0.3\right),\left(x_{2}, 0.2,0.2\right)\right.$, $\left.\left(x_{3}, 0.1,0.1\right)\right\}$ is given. To interpret the notions of three patterns, we borrow the idea of Wang and Xin [19]. Given three minerals of mineral fields, each is featured by the content of three minerals and contains one kind of typical hybrid minerals. The three kinds of typical hybrid minerals are represented by IFSs $A_{1}, A_{2}, A_{3}$ in $X$, respectively. Given another kind of hybrid mineral $B$, to which field does this kind of mineral $B$ most probably belong to ? By (1)-(4), we have

$$
\begin{aligned}
& S_{1}\left(A_{1}, B\right)=1.000, S_{1}\left(A_{2}, B\right)=0.877, S_{1}\left(A_{3}, B\right)=0.600, \\
& S_{2}^{2}\left(A_{1}, B\right)=1.000, S_{2}^{2}\left(A_{2}, B\right)=0.847, S_{2}^{2}\left(A_{3}, B\right)=0.568, \\
& S_{3}\left(A_{1}, B\right)=1,000, S_{3}\left(A_{2}, B\right)=0.765, S_{3}\left(A_{3}, B\right)=0.429, \\
& S_{4}^{2}\left(A_{1}, B\right)=1,000, S_{4}^{2}\left(A_{2}, B\right)=0.719, S_{4}^{2}\left(A_{3}, B\right)=0.397 .
\end{aligned}
$$


From this data, it is evident that $A_{1}=B$. That, is the sample $B$ belong to the pattern $A_{1}$.

Example 2. [13] Assume that there are three patterns denoted with IFSs in $X=\left\{x_{1}, x_{2}, x_{3}\right\}$. The three patterns are denoted as follows:

$$
\begin{aligned}
& A_{1}=\left\{\left(x_{1}, 0.3,0.3\right),\left(x_{2}, 0.2,0.2\right),\left(x_{3}, 0.1,0.1\right)\right\} \\
& A_{2}=\left\{\left(x_{1}, 0.2,0.2\right),\left(x_{2}, 0.2,0.2\right),\left(x_{3}, 0.2,0.2\right)\right\} .
\end{aligned}
$$

Assume that a sample $B=\left\{\left(x_{1}, 0.3,0.3\right),\left(x_{2}, 0.2,0.2\right)\right.$, $\left.\left(x_{3}, 0.1,0.1\right)\right\}$ is given. By, we have

$$
\begin{aligned}
& S_{1}\left(A_{1}, B\right)=0.833, S_{1}\left(A_{2}, B\right)=0.733, \\
& S_{2}^{2}\left(A_{1}, B\right)=0.827, S_{2}^{2}\left(A_{2}, B\right)=0.727, \\
& S_{3}\left(A_{1}, B\right)=0.714, S_{3}\left(A_{2}, B\right)=0.579, \\
& S_{4}^{2}\left(A_{1}, B\right)=0.705, S_{4}^{2}\left(A_{2}, B\right)=0.559 .
\end{aligned}
$$

Based on the above results, it is seen that the sample $B$ belongs to the pattern $A_{1}$ according to the principle of the maximum degree of similarity between IFSs. This classification result is different from Liang and Shi [13], where their measure $S_{s}^{p}$ cannot classify this sample because of $S_{s}^{p}\left(A_{1}, B\right)=S_{s}^{p}\left(A_{2}, B\right)$.

\section{Acknowledgements}

This study was supported by research funds from Dong-A University.

\section{References}

[1] K. Atanassov, "Intuitionistic fuzzy sets," Fuzzy Sets and Systems, vol. 20 pp. 87-96, 1986.

[2] K. Atanassov, "More on intuitionistic fuzzy sets," Fuzzy Sets and Systems, vol. 33, pp. 37-46, 1989.

[3] K. Atanassov, "New operations defined over the intuitionistic fuzzy sets," Fuzzy Sets and Systems, vol. 61, pp. 137-142, 1994.

[4] k. Atanassov, g. Gargov, "Intuitionistic fuzzy logic," CR Acad. Bulg. Soc. vol. 43, pp. 9-12, 1990.

[5] H. Bustince, P. Burillo, "Vague sets are intuitionistic fuzzy sets," Fuzzy Sets and Systems, vol. 79, pp. 403405, 1996.

[6] S.M. Chen, "Similarity measure between vague sets and elements," IEEE Trans. Systems Man Cybernt., vol. 27, pp. 153-158, 1997.
[7] S.M. Chen, J.M. Tan, "Handling multi-criteria fuzzy decision-making problems based on vague sets," Fuzzy Sets and Systems, vol. 67, pp. 163-172, 1994.

[8] S.K. De, P. Biswas, A.R. Roy, "An application of intuitionistic fuzzy sets in medical diagnosis," Fuzzy Sets and Systems, vol. 117, pp. 209-213, 2001.

[9] W.L. Gau, D.J. Buehere, "Vague sets," IEEE Trans. Systems Man Cybernt., vol. 23, pp. 610-614, 1994.

[10] W.L. Hung, M.S. Yang, "Similarity measures of intuitionistic fuzzy sets based on Hausdorff distance," Pattern Recognition Lett., vol. 25, pp. 1603-1611, 2004.

[11] D. Li, C. Cheng, "New similarity measures of intuitionistic fuzzy sets and applications to pattern recognitions," Pattern Recognition Lett., vol. 23, pp. 221225, 2002.

[12] Y. Li, D.L. Olson, Z. Qin, "Similarity measures between intuitinistic fuzzy (vague) sets: A comparative analysis," Pattern Recognition Lett., vol. 28, pp. 278285, 2007.

[13] Z. Liang, P. Shi, "Similarity measures on intuitionistic fuzzy sets," Pattern Recognition Lett., vol. 24, pp. 2687-2693, 2003.

[14] H.B. Mitchell, "On the Dengfeng-Chuitian similarity measure and its application to pattern recognition," Pattern Recognition Lett., vol. 24, pp. 3101-3104, 2003.

[15] E. Szmidt, "Applications of intuitionistic fuzzy sets in decision making," (D Sc dissertation) Tech. Univ, Sofia 2000.

[16] E. Szmit, J. Kacprzyk, "Distances between intuitionistic fuzzy sets", Fuzzy Sets and Systems, vol. 114, pp. 505-518, 2000.

[17] E. Szmit, J. Kacprzyk, "A new concept of a similarity measure for intuitionistic fuzzy sets and its use in group decision making", LNAI vol. 3558, pp. 272$282,2005$.

[18] I.K. Vlachos, G.D. Sergiadis, "Intuitionistic fuzzy information - Applications to pattern recognition," Pattern Recognition Lett., vol. 28, pp. 197-206, 2007.

[19] W. Wang, X. Xin, "Distance measure between intuitionistic fuzzy sets," Pattern Recognition Lett., vol. 26, pp. 2063-2069, 2005.

[20] L.A. Zadeh, "Fuzzy sets." Inform and Control, vol. 8, pp. 338-353, 1965. 
[21] Zadeh LA, Fuzzy sets, Inform. and Control, 8 (1965) 338-353.

\section{Jin Han Park}

Professor of Pukyong National University

Research Area: Fuzzy mathematics, Fuzzy topology,

General topology, Decision making

E-mail : jihpark@pknu.ac.kr
Ki Moon Lim

Lecturer of Pukyong National University

Research Area: Fuzzy topology, General topology

E-mail : ulve@hanmail.net

\section{Young Chel Kwun}

Professor of Dong-A University

Research Area: Fuzzy mathematics, Fuzzy differential equations, Decision making

E-mail : yckwun@dau.ac.kr 